This item was submitted to Loughborough's Research Repository by the author.

Items in Figshare are protected by copyright, with all rights reserved, unless otherwise indicated.

\title{
Step sequence and direction detection of four square step test
}

PLEASE CITE THE PUBLISHED VERSION

http://dx.doi.org/10.1109/LRA.2017.2723929

PUBLISHER

(c) IEEE

VERSION

AM (Accepted Manuscript)

LICENCE

CC BY-NC-ND 4.0

REPOSITORY RECORD

Kong, Weisheng, Lauren Waaning, Salvatore Sessa, Massimiliano Zecca, Daniele Magistro, Hikaru Takeuchi, Ryuta Kawashima, and Atsuo Takanishi. 2019. "Step Sequence and Direction Detection of Four Square Step Test”. figshare. https://hdl.handle.net/2134/25962. 


\title{
Step Sequence and Direction Detection of Four Square Step Test
}

\author{
W. Kong ${ }^{1}$, L. Wanning ${ }^{2}$, S. Sessa ${ }^{1}$, M. Zecca ${ }^{2,3}$, \\ D. Magistro ${ }^{3,4}$, H. Takeuchi ${ }^{6}$, R. Kawashima ${ }^{6}$, and A. Takanishi ${ }^{7,8}$
}

\begin{abstract}
Poor balance control and falls are big issues for older adults that due to aging decline have a lower postural balance and directional control in balance performance than younger age groups. The four square step test (FSST) was developed to evaluate rapid stepping that is often required when changing direction and avoiding obstacles while walking. However, previous researchers used only the total time as the assessment in the test. The aim of this paper is to objectively quantify the sequence and direction of the steps in FSST, by using two inertial sensors placed on both feet. An algorithm was developed to automatically segment the steps performed during the test, and calculate the stepping direction from the linear velocity of the foot. Experiments were conducted with 100 Japanese healthy older adults, where sensor data and video of 20 subjects were randomly subtracted for algorithm verification. The results showed that the algorithm succeeded for $\mathbf{7 1 . 7 \%}$ trials in recognizing both the step sequence and step direction in FSST, while $90.2 \%$ of the detection failure could be excluded with an auto verification method.
\end{abstract}

Index Terms-Automation in life sciences: biotechnology, pharmaceutical and health care; health care management; sensor fusion.

\section{INTRODUCTION}

$\mathbf{T}$ HE population of older adults is growing faster than any other age group worldwide [1]. Older adults have a lower postural balance and a lower directional control in balance performance than younger age groups [2]. With increased age,

Manuscript received: Februray, 15, 2017; Revised May, 30, 2017; Accepted June, 18, 2017.

This paper was recommended for publication by Editor W. K. Chung upon evaluation of the Associate Editor and Reviewers' comments. *This research has been supported by the JSPS Grant-in-Aid for Young Scientists (Wakate B) [25750259] and [15K21437]. The present work was also supported in part by the Program for Leading Graduate Schools, "Graduate Program for Embodiment Informatics" of the Ministry of Education, Culture, Sports, Science and Technology. This research has also been partially supported by the UK-HEFCE Catalyst grant and by the LU-EESE startup grant.

${ }^{1}$ Graduate School of Advanced Science and Engineering, Waseda University, Tokyo, Japan.

${ }^{2}$ Wolfson School of Mechanical, Electrical and Manufacturing Engineering, Loughborough University, UK.

${ }^{3}$ National Centre for Sport and Exercise Medicine, UK.

${ }^{4}$ School of Sport, Exercise, and Health Sciences, Loughborough University, $\mathrm{UK}$.

${ }^{5}$ NIHR Leicester-Loughborough Diet, Lifestyle and Physical Activity Biomedical Research Unit, UK

${ }^{6}$ Department of Functional Brain Imaging. IDAC, Tohoku University, Sendai, Japan.

${ }^{7}$ Department of Modern Mechanical Engineering, Waseda University, Tokyo, Japan.

${ }^{8}$ Humanoid Robotics Institute (HRI), Waseda University, Tokyo, Japan. contactetakanishi.mech.waseda.ac.jp

Digital Object Identifier (DOI): see top of this page. there is a progressive loss of functioning of these systems which can contribute to balance deficits [3] and risk of fall.

Therefore, it is important to assess the balance capabilities of older adults to track aging decline and eventually prevent the falling events. The 'Four Square Step Test' (FSST) is a possible test to assess dynamic balance. In particular, this test assesses the dynamic balance ability when stepping in four directions: forwards, backwards, leftwards, and rightwards [4]. Such stepping tasks are highly relevant to the activities of daily living [5], which is confirmed from the feedback of the participants [6]. In the medical application, FSST showed good sensitivity and specificity to distinguish the multiple and nonmultiple fallers [5]. Furthermore, its functionality has been confirmed in patients with stroke [6], [7], Parkinson's disease [8], and vestibular disorders [9].

According to the protocol of FSST [4], the only quantitative assessment in FSST is the total time to successfully complete the test [4], which is measured by an operator with a stopwatch. Consequently, the trials must be discarded if their motions differ from the protocol. Currently, the assessment of FSST is only based on the successful trials, while the failed trials are noted and excluded.

However, FSST is particularly challenging and prone to mistakes. According to Blennerhassett et al., 40-62\% of ambulant poststroke participants failed at least once within 2 FSST trials [6]. Goh et al. also reported that $20 \%$ of the participants with stroke had unsuccessful trials within 3 FSST trials [7]. Participants have to repeat when failure, but if the number of successful trials is not enough after certain times of attempts, the assessment for that participant is failed. Assessment only on the successful trials increases the number of trials and even leads to assessment failure. Furthermore, the underlying problem would be more easily understood through the mistakes in the unsuccessful trials, rather than the successful ones.

To solve this problem, more indicators about the performance in FSST are needed, which can be achieved through segmenting the test into different stepping phases. In this way, not only a more detailed assessment can be obtained compared with total time only, but also the unsuccessful trials can be evaluated. To lay the foundation for assessment in each stepping phase, this research proposed algorithms for auto step segmentation and step direction detection in FSST.

The step sequence and direction can be detected in an ecological setting with either marker-less optical motion capture such as Microsoft Kinect [10], image processing with camera [11], or with inertial sensors [12]. The advantage of inertial 
sensors is less limitation on working space. Both camera and Kinect are affected by illumination and require a larger space for keeping a distance between the device and participant. Furthermore, the upper body sway measured by additional inertial sensors on the trunk and/or head is more precise and much easily to be synchronized with step segmentation.

The first challenge for step segmentation in FSST is that the algorithm must be applicable for forwards, backwards, and sideways stepping. In the field of mobility test assessment with inertial sensors, step segmentation algorithms are designed for forwards stepping, or more precisely walking. Furthermore, the kinematic pattern of walking is utilized in most of these algorithms [12], [13], which improves the segmentation precision and accuracy but also limits their application on other directions, because the patterns are different. The solution is to use the algorithms which segment steps by identifying the static and non-static periods in a data series, for example the ones thresholding on foot acceleration variance [14] and foot angular velocity magnitude [15]. These algorithms are originally intended to cancel the velocity drifting in pedestrian navigation, but can also be applied to backwards and sideways stepping.

The second challenge is to segment the multiple continuous steps of one leg, while the other leg keeps static. This is a unique challenge in FSST, as in walking both legs always interchange their roles (stance/swing leg) after each step. To the knowledge of the authors, we didn't find such algorithms in the studies of mobility test assessment. We found related studies in dancing [16] which recognized dance pattern from inertial sensors through comparing the similarities of two blocks of motion with dynamic time wrapping. But it requires either a large set of training data or a set of well-defined sample motion data.

Therefore, this paper aims to overcome the two challenges. Specifically, the objective is to propose the algorithm of step segmentation and step direction detection during the FSST, and then verify the results with manual segmentation with the video of the experiment. Compared with the state of the art, the proposed algorithm adds the capability to segment multiple continuous steps with the same leg, while keeping the simplicity for not requiring training or sample motion data. Moreover, the proposed method provides a detection for forwards, backwards, and sideways stepping direction.

\section{MATERIALS AND METHODS}

\section{A. Experimental Setup}

40 male and 60 female healthy older adult volunteers ranging from 65 to 76 years old (age $=68.8 \pm 3.2$ years old) were recruited at Institute of Development, Aging and Cancer, Tohoku University, Sendai, Japan. The experiments were approved by the ethical committees of Waseda University and Tohoku University. A subset of 20 subjects were randomly extracted from the database for algorithm verification.

Participants were required to rapidly change direction while stepping forward, backward and sideways, in a predetermined sequence, over an indicated area placed in a cross configuration on the ground (see Fig. 1). The participant's starting position is in the top-left corner of the cross. Then, the participant starts by stepping to the right, backward to the left and forward, into each quadrant in the clockwise direction, followed by the reverse sequence in the counter-clockwise direction.

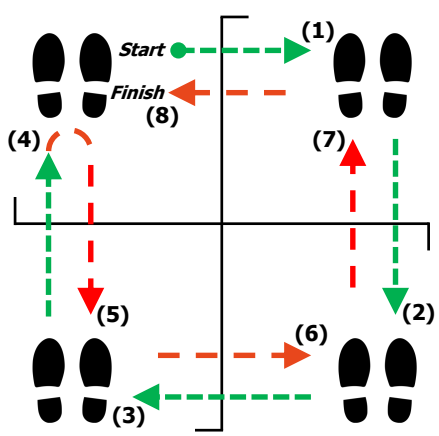

Fig. 1. Stepping sequence in Four Square Step Test (FSST). The green dash arrows show the clockwise steps from (1) to (4), while the red dash arrows show the counter-clockwise steps from (5) to (8).

Before the experiment, participants were required to remember the step sequence and practice until they could perform the exercise correctly. After that, the test started in the following three conditions:

1) Single-task: FSST;

2) Dual-task: FSST while back counting by 7 from a random number between 90 and 100;

3) Multi-task: FSST while back counting plus holding a cup of water.

Each condition was repeated for three times. The dual-task and multi-task conditions were included because these conditions better simulate the scenario in everyday life, where the mobility tasks are rarely carried out alone. The mobility tasks, such as stepping in various directions in the case of FSST, are often associated with one or even more additional activities, such as talking or paying attention to the environment [17]. These additional activities might lead to increased variabilities in a range of spatio-temporal stepping parameters [18].

\section{B. Sensor System and Placement}

The sensor named WB-4R (Waseda bioinstrumentation 4R) was used in the experiment. WB-4R is an Inertial Measurement Unit (IMU) containing a tri-axial accelerometer, a triaxial gyroscope and a tri-axial magnetometer embedded in a compact package $(17 \times 20 \times 8 \mathrm{~mm}, 3.9 \mathrm{~g}$, sampling frequency $200 \mathrm{~Hz}$ ). WB-4R could be tightened on the human body through an elastic band with a button and slots (Fig. 2(a)), which allows an easy, fast, and adaptable wearing of the device on the participants. All the WB-4R sensors are synchronized through CAN bus via wired connection to a central board. More detailed characteristics of the sensor could be found in [12]. In this research, two WB-4R sensors were placed on the frontal side of both feet, see Fig. 2(b). 


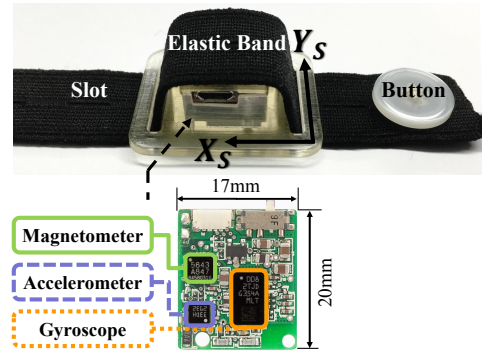

(a) WB-4R IMU.

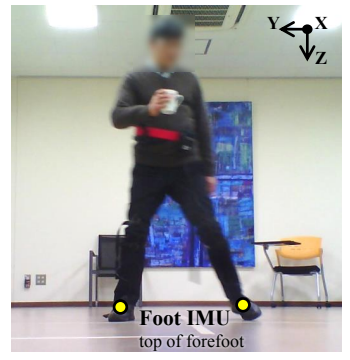

(b) Sensor placement.
Fig. 2. Inertial Measurement Unit (IMU) sensor system.

\section{Step Segmentation}

Measurements from IMUs on feet were used for the event detection because their signal showed a clear difference between stance phase and swing phase.

The modulus of foot angular velocity is first calculated,

$$
\Omega_{i, j}=\left\|\omega_{i, j}\right\| \quad i \in\{1, \ldots, N\}, j \in\{0,1\}
$$

where $i$ representing the sample frame number, $j$ representing the right $(j=0)$ and left foot $(j=1)$, see Fig. 4(a). Then, $\Omega_{i, j}$ is thresholded to find the non-static moments. In particular, if the variable of one foot is higher than the variable of the other foot and higher than a the preset threshold, it is marked as 1 . Equation (2) shows the thresholding for the right foot $(j=0)$. The similar equation is used for the left foot. Here, the purpose of setting a preset threshold is to reject the case that both feet are static. We selected the preset threshold at $30 \mathrm{deg} / \mathrm{s}$, which is about 20 times larger than the 95 percentile of the angular velocity when the sensor is placed statically on a table (about $1.4 \mathrm{deg} / \mathrm{s})$.

$$
b_{i, 0}= \begin{cases}1, & \Omega_{i, 0}>\Omega_{i, 1} \text { and } \Omega_{i, 0}>30 \mathrm{deg} / \mathrm{s} \\ 0, & \text { otherwise }\end{cases}
$$

Furthermore, merging and discarding of the periods are performed separately for each foot. The purpose is to prevent any short change in angular velocity, for example during foot strike, from affecting the result. Morphological closing (operator $\bullet$ in Equation (3)) merges the periods with an interval in between shorter than 100 milliseconds, and morphological opening (operator $\circ$ in Equation (3)) discards the periods shorter than 100 milliseconds. With a sampling rate at $200 \mathrm{~Hz}$, 100 milliseconds corresponds to 20 sample points.

$$
\mathbf{B}=\left(\mathbf{b} \bullet \mathbf{1}_{1 \times 20}\right) \circ \mathbf{1}_{1 \times 20}
$$

Here we omit the subscript $j$ which indicates the foot from the equations, since Equation (3) and all the following operations are performed separately on each foot. $\mathbf{b}=\left[b_{1}, b_{2}, \ldots, b_{N}\right]$ represents the time series of results from Equation 2. B is a binary vector which consists of a serial of continuous $\mathbf{0}$ or 1 sub-vectors, where the $\mathbf{0}$ sub-vectors represent the potential static phases and the 1 sub-vectors represent the potential nonstatic phases, see Fig. 4(b).

The potential static and non-static phases do not necessarily correspond with the stance and swing phases in FSST. We observed that during the double stance phase, subjects may rotate their stance toes or even shortly shift their stance foot medially preparing for the next stride. Also, we observed that subjects may slightly lift and land their foot for multiple times at the same place, when considering which leg should leave first or which direction should go for the next stride. Moreover, unlike walking in a straight line, in FSST one leg can move multiple continuous steps. As shown in Fig. 3(a), when moving backwards (2nd stride) and then to the left (3rd stride), the left foot may move continuously for two steps, if the right foot leaves first when stepping backwards. Besides, after finishing the four clockwise strides, subjects may make mistake stepping their right foot to the right, and suddenly realize the mistake and put the right leg back, in the extreme case shown in Fig. 3(b) the subject may move 4 continuous right steps with almost zero stance phase in between.

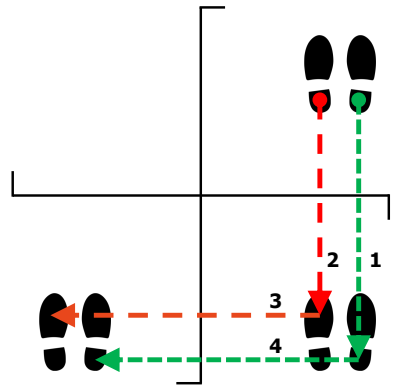

(a) 2 continuous left steps.

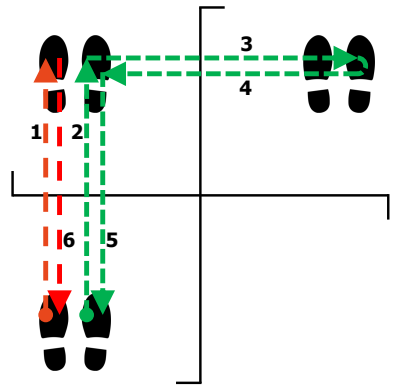

(b) 4 continuous right steps.
Fig. 3. Example of multiple continuous steps of the same foot. The red dash arrows represent the movement of left foot, while the green dash arrows represent the right foot. The number of each arrow shows the sequence of the step within the example.

To understand whether a non-static phase is a foot rotation/shift at the same place, a swing phase, or multiple swing phases, we want to check the distance traveled by the foot and if there are multiple peaks in the horizontal linear velocity.

Foot velocity is calculated by integrating the foot acceleration which is with respect to the human body coordinates frame. The acceleration measured by IMU is with respect to the sensor coordinates frame. Therefore changing reference coordinates frame must be performed before the integration. The relative rotation between the sensor coordinates frame and human body coordinates frame is calculated through extended Kalman filter (EKF) which turns angular velocity and acceleration into quaternion $q$ (Equation (4)). Equation (5) uses the generated quaternion to change the reference coordinates frame of acceleration from IMU sensor frame $\{I\}$ to Global frame $\{G\}$.

$$
\begin{aligned}
& { }_{G}^{I} \mathbf{q}_{i}=E K F\left({ }^{I} \omega_{i},{ }^{I} \ddot{\mathbf{x}}_{i}\right) \\
& {\left[0,{ }^{G} \ddot{\mathbf{x}}_{i}\right]^{T}={ }_{G}^{I} \mathbf{q}_{i}^{-1}\left[0,{ }^{I} \ddot{\mathbf{x}}_{i}\right]^{T}{ }_{G}^{I} \mathbf{q}_{i}}
\end{aligned}
$$

where ${ }^{I} \omega_{i}$ and ${ }^{I} \ddot{\mathbf{x}}_{i}$ represent the angular velocity and linear acceleration with respect to $\{I\}$, while the ${ }_{G}^{I} \mathbf{q}_{i}$ is the quaternion rotating from $\{G\}$ to $\{I\}$, and finally ${ }^{G} \ddot{\mathbf{x}}_{i}$ represents linear acceleration with respect to $\{G\}$. In the following part of the paper, we omit the superscript for coordinates frame and all the variables are with respect to $\{G\}$. 
Then the linear velocity is obtained by integrating the linear acceleration during the non-static phase. Taken the non-static phase starting and ending at the $m$ and $n$ sample frames respectively, the integration starts from zero vector and accumulates with time (Equation (6)).

$$
\dot{\mathbf{x}}_{i}^{\prime}= \begin{cases}{[0,0,0]^{T},} & i=m \\ \dot{\mathbf{x}}_{i-1}^{\prime}+\ddot{\mathbf{x}}_{i-1} / f_{s}, & m<i \leq n\end{cases}
$$

where $f_{s}$ represents the sampling frequency. Because the integration of one non-static phase starts and ends when the foot is static, the velocity should be zero at both ends of integration. However, the bias in acceleration causes the integration to drift linearly with time. In Equation (7), the linear drift is estimated by linear interpolation between velocities at the first and last samples of the non-static phase and then removed from velocity.

$$
\dot{\mathbf{x}}_{i}=\dot{\mathbf{x}}_{i}^{\prime}-\left(\dot{\mathbf{x}}_{m}^{\prime}+\frac{\dot{\mathbf{x}}_{n}^{\prime}-\dot{\mathbf{x}}_{m}^{\prime}}{n-m}(i-m)\right)
$$

Finally, the distance is calculated by integrating the linear velocity as shown in Equation (8) below.

$$
\mathbf{x}_{i}= \begin{cases}{[0,0,0]^{T},} & i=m \\ \mathbf{x}_{i-1}+\dot{\mathbf{x}}_{i-1} / f_{s}, & m<i \leq n\end{cases}
$$

With the velocity and distance calculated, we use them to determine whether a non-static phase is a foot rotation/shift at the same place, a single swing phase, or multiple swing phases.

To do that, we first inspect if there are multiple swing phases in one non-static phase, by checking if there is more than one main peak in the foot linear velocity, see Fig. 4(c). The main peak roughly represents the part of a swing phase when the foot is moving fast. The main peak is defined as a continuous series of data which are over a certain velocity, and the length of the data is at least 100 milliseconds. Specifically, we empirically selected the median value in each non-static phase as its threshold for foot velocity,

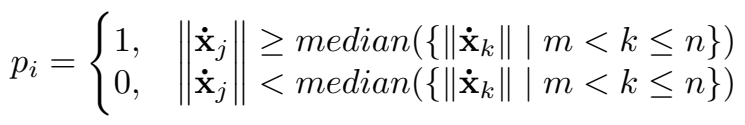

where $i=j-m+1, m<j \leq n$. The reason to choose the median is to adapt to the various stepping velocities of different participants and in different directions, while being robust against the extreme value caused by foot strike. Then we remove the peaks shorter than $100 \mathrm{~ms}$ with the morphological opening, followed by removing the intervals shorter than $100 \mathrm{~ms}$ using the morphological closing, see Equation (10).

$$
\mathbf{P}=\left(\mathbf{p} \circ \mathbf{1}_{1 \times 20}\right) \bullet \mathbf{1}_{1 \times 20}
$$

If there is only one peak, or in another word, $\mathbf{P}$ contains only one continuous 1 vector, the non-static phase is kept as it is. However, if there are multiple velocity peaks, the middle points of the intervals between the peaks are used to separate the peaks and therefore separate the non-static phase. For example: $\mathbf{P}=\left[\mathbf{0}_{1 \times L_{0}}, \mathbf{1}_{1 \times L_{1}}, \mathbf{0}_{1 \times L_{2}}, \mathbf{1}_{1 \times L_{3}}, \mathbf{0}_{1 \times L_{4}}\right]$ where there are two peaks $\left(\mathbf{1}_{1 \times L_{1}}\right.$ and $\left.\mathbf{1}_{1 \times L_{3}}\right)$ with length $L_{1}$ and $L_{3}$ respectively. The middle point of the interval $\mathbf{0}_{1 \times L_{2}}$ is then used to separate the two peaks. Suppose the index of the middle point is $i=m-1+L_{0}+L_{1}+\operatorname{round}\left(L_{2} / 2\right)$, the corresponding element of $\mathbf{B}$ in Equation (3) should be set to zero $B_{i}=0$, see Fig. 4(d).

To identify whether a non-static phase is a foot rotation/shift at the same place, we calculated the step distance and checked if it is shorter than a certain threshold. Suppose one non-static phase starts and stops at $m$ and $n$ sample frames respectively, the step length is calculated by finding the horizontal distance between the $\mathbf{x}_{m}$ and $\mathbf{x}_{n}$.

$$
d=\left\|\left(\mathbf{x}_{n}-\mathbf{x}_{m}\right) \cdot[1,1,0]^{T}\right\|
$$

If the step length $d$ is shorter than $10 \mathrm{~cm}$, we think the nonstatic phase is a foot rotation/shift at the same place, therefore exclude it from the following analysis.
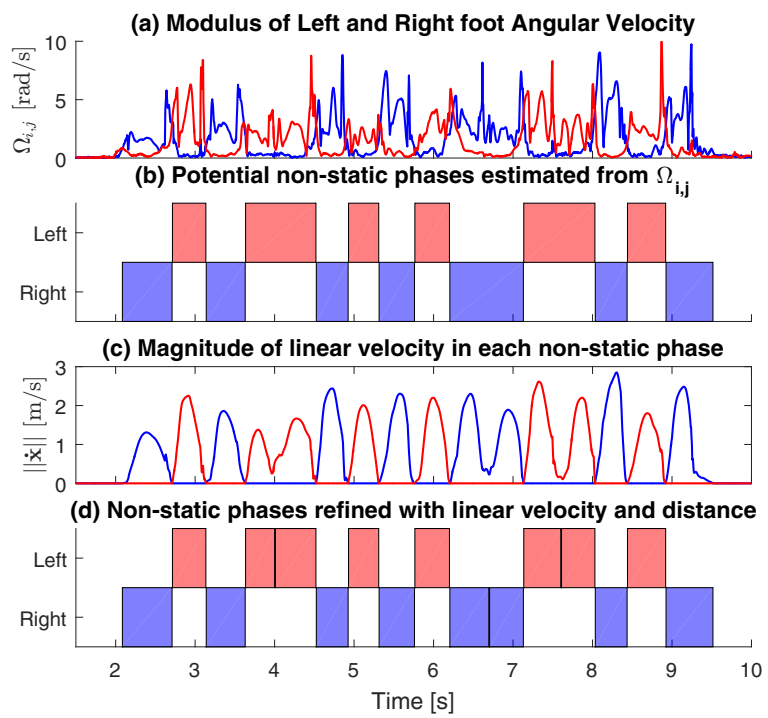

Fig. 4. Step segmentation. (a) shows the angular velocity modulus of the left foot (red line) and right foot (blue line), the result of Equation (1). The foot with higher value is more likely to be non-static; (b) shows the potential non-static phases estimated based on (a) with Equation (3); (c) shows the magnitude of the linear velocity calculated in Equation (7); (d) shows the final non-static phases refined, which uses the linear velocity and distance to divide multiple continuous steps.

\section{Step direction detection}

The Cartesian coordinates are transformed into cylindrical coordinates and since we are interested in the horizontal movement we keep only the angle $\theta$ and radius $\mathbf{r}$ :

$$
\left\{\begin{array}{l}
\theta_{i}=\operatorname{atan}\left(\dot{x}_{x, i}, \dot{x}_{y, i}\right) \\
r_{i}=\sqrt{\dot{x}_{x, i}^{2}+\dot{x}_{y, i}^{2}}
\end{array}\right.
$$

where $m \leq i \leq n$. The angle representing the direction of one stride is taken at the maximum linear velocity point:

$$
\Theta=\theta_{k}, \quad k=\operatorname{argmax}\left(r_{i}\right), \quad m \leq i \leq n
$$

$\Delta \Theta$, the change in direction between strides, is then calculated and wrapped to $\pm \pi$. Compared to the angle $\Theta, \Delta \Theta$ is more 
robust to error introduced by sensor misplacement, and also to the angular drift around the vertical axis.

$$
\Delta \Theta_{i}=\Theta_{i+1}-\Theta_{i}
$$

Here, $i$ represents the number of step for one foot, for example $i=8$ means the 8 th step.

TABLE I

STEPPING DIRECTION AND LABEL IN CORRECT SEQUENCE OF FSST.

\begin{tabular}{lllllllll}
\hline Step No. (i) & 1 & 2 & 3 & 4 & 5 & 6 & 7 & 8 \\
\hline Direction & $\rightarrow$ & $\downarrow$ & $\leftarrow$ & $\uparrow$ & $\downarrow$ & $\rightarrow$ & $\uparrow$ & $\leftarrow$ \\
Label of direction $\left(D_{i}\right)$ & 0 & 1 & 2 & 3 & 1 & 0 & 3 & 2 \\
Change in direction & - & $\mathrm{R}$ & $\mathrm{R}$ & $\mathrm{R}$ & $\mathrm{B}$ & $\mathrm{L}$ & $\mathrm{L}$ & $\mathrm{L}$ \\
\hline
\end{tabular}

R: turn Right; B: turn Back; L: turn Left.

Finally, the direction of the stride is determined from $\Delta \Theta$, as shown in Equation 15. Stepping to the right, back, left and front is labeled as 0,1,2 and 3 respectively, and the direction of the first stride is set to zero $D_{1}=0$, as shown in Table I.

$$
D_{i+1}= \begin{cases}\bmod \left(D_{i}-1,4\right), & 0 \leq \Delta \Theta_{i} \leq \frac{3 \pi}{4} \\ \bmod \left(D_{i}+1,4\right), & -\frac{3 \pi}{4} \leq \Delta \Theta_{i}<0 \\ \bmod \left(D_{i}-2,4\right), & \text { otherwise }\end{cases}
$$

The thresholds are determined by segmenting the angle into four areas, marked as A, B, C and D in Fig. 5. However, area $\mathrm{A}$ should be removed because stepping without changing direction is not allowed by the protocol of FSST. The positive half of area $\mathrm{A}$ is then combined with area $\mathrm{B}$ to form the thresholds $0 \leq \Delta \Theta_{i} \leq \frac{3 \pi}{4}$, where the direction of the next step is changing towards the left side of the current step, during counter-clockwise steppings. The negative half of area $\mathrm{A}$ is combined with area D to form the thresholds $-\frac{3 \pi}{4} \leq \Delta \Theta_{j}<0$, where the direction of the next step is changing towards the right side of the current step, during clockwise steppings. Area $\mathrm{C}$ forms the thresholds $-\pi \leq$ $\Delta \Theta_{j}<-\frac{3 \pi}{4}$ and $\frac{3 \pi}{4}<\Delta \Theta_{j} \leq \pi$, where the direction of the next step is changing towards the back side of the current step, when switching from clockwise to counter-clockwise, or vice versa.

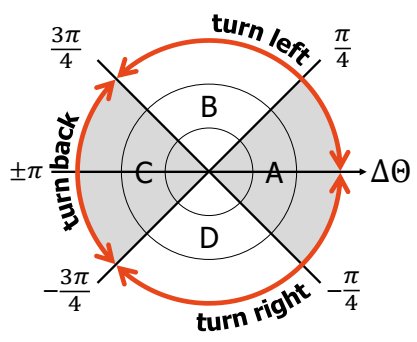

Fig. 5. Thresholds for stepping direction. A, B, C, and D mark four 90 degrees areas centered at $0,90,180$ and -90 degrees. The zero degree represents the direction of the current step. The three red arrows show the thresholds for determining step directions in Equation (15). The "turn left", "turn back", and "turn right" on the red arrows present the change in direction from the current to the next step.

\section{E. Automatic check of detection failure}

After the step segmentation and step direction detection, an automatic check is run to verify if the step sequence and the step direction are possible. The check items are as follows:

1) The subject should only move between the 4 corners of the square;

2) Both feet should be at the same corner after each foot has moved for one or multiple successive steps.

The position of the foot, more specifically the corner at which the foot lands, is calculated by the accumulating the direction of each step.

\section{RESULTS}

To verify the step sequence and direction algorithm, all the 180 trials were manually checked by watching the experiment video, with the following check items:

1) SEQuence of left and right feet (SEQ);

2) Right foot stepping DIRection (RDIR);

3) Left foot stepping DIRection (LDIR),

Overall, in a total of 180 trials, 139 trials (77.2\%) succeeded in the step sequence segmentation, and 129 trials $(71.7 \%)$ met all the three requirements, as shown in Table II.

TABLE II

CORRECT RATE FOR ALL THE 180 TRIALS.

\begin{tabular}{lcccc}
\hline & SEQ & RDIR & LDIR & All correct \\
\hline Successed trials & 139 & 148 & 155 & 129 \\
Percentage & $77.2 \%$ & $82.2 \%$ & $86.1 \%$ & $71.7 \%$ \\
\hline
\end{tabular}

The automatic failure check, as shown Table III, successfully found 46 from all the 51 failures (specificity: 90.2\%) and showed excellent sensitivity $(100 \%)$. The automatic verification was precise even compared with the manual verification. In fact, when double checking the unmatched results between auto and manual, we also found manual errors in 5 trials.

TABLE III

RESULT OF AUTOMATIC FAILURE CHECK.

\begin{tabular}{llll}
\hline & & \multicolumn{2}{c}{ Predicted } \\
& Positive & Negative \\
\hline \multirow{2}{*}{ True } & Positive & 129 & 0 \\
& Negative & 5 & 46 \\
\hline
\end{tabular}

\section{DIscussion}

\section{A. Step Sequence Segmentation}

The major source of error was the step sequence segmentation. First, it caused most failures: 41 out of 180 trials; second, it is the basis of step direction detection in our algorithm. If the step segmentation goes wrong, it would lead to errors in step direction detection. In fact, within the 41 SEQ failed trials, less than half succeeded in finding the correct direction of a single leg (RDIR: 16, LDIR: 20), and none succeeded for RDIR and LDIR at the same time.

To understand the source of error, we matched the auto and manually detected step sequence. We found that the step 
sequence segmentation performed well at the step level. Within total 2954 steps in 180 trials, 2902 steps are correctly detected. The sensitivity is $99.3 \%$, while the Positive Predictive Value (PPV) reaches $98.9 \%$.

For the 52 unmatched steps, as shown in Table IV, nearly half of them ( 25 steps) happen at the beginning and the end of the step sequence, while the other 27 steps occur in the middle of the test. The false positives at the beginning or end of the test are caused by including steps outside the test period, for example the posture adjustment before the test begins. The false negatives at the beginning or end of the test are caused by losing the first/last step, mainly because of the relatively slow motion or early finished data logging.

TABLE IV

FAILURE AT THE BEGINNING AND END OF STEP SEQUENCE.

\begin{tabular}{lllll}
\hline & All & Begin & Middle & End \\
\hline False negative & 20 & 3 & 13 & 4 \\
False positive & 32 & 11 & 14 & 7 \\
\hline
\end{tabular}

\section{B. Step Direction Detection}

The correct rate of step direction is higher compared with the step segmentation. Within the 139 trials where step segmentation is correct, 129 trials $(92.8 \%)$ succeed in finding step directions for both feet, as shown in Table V.

TABLE V

CORRECT RATE OF STEP DIRECTION DETECTION FOR THE 139 TRIALS WITH STEP SEQUENCE CORRECTLY DETECTED.

\begin{tabular}{lccc}
\hline & RDIR & LDIR & RDIR \& LDIR \\
\hline Successed trials & 132 & 135 & 129 \\
Percentage & $95.0 \%$ & $97.1 \%$ & $92.8 \%$ \\
\hline
\end{tabular}

Within the 10 failed trials, we found that 5 of them were due to the unexpected movement of the subjects. Specifically, one trial was failed because the subject started the test by stepping backwards, walking two times counterclockwise, see Fig. 6(a). However, in our algorithm, the first step was initialized to be towards the right. Besides, four trials were failed because the subject stepped in the diagonal direction. As shown in Fig. 6(b), after finishing the first four clockwise steps, these subjects should step backwards to start four counterclockwise steps. However, they made mistake by moving their right foot to the right. After landing their right foot, they realized that the direction was wrong and moved the right foot directly along the diagonal to the back of the body. Such cases cannot be handled by our algorithm, so physicians or analysts should perform manual step direction recognition once noticing them during the experiment.

Concerning the step level, within the 2282 steps (left: 1137; right: 1145$)$ of the 139 correctly segmented trials, the direction of 47 steps $(2.1 \%)$ from 10 trials was wrong. This number, however, overestimates the real times of error, because in the algorithm the direction of one step is determined based on the direction of the previous step. Therefore, an error in one

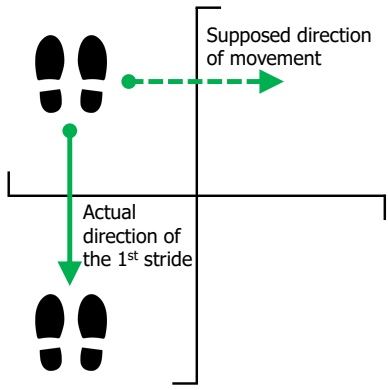

(a) Wrong first stride.

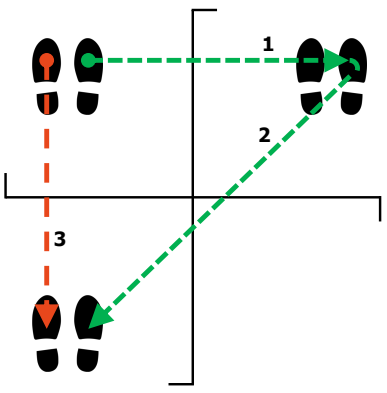

(b) Diagonal step.
Fig. 6. Unexpected movements which caused error in step detection.

step could lead to errors in all following steps. After removing the error caused by previous steps, we found 13 errors in the failed 47 steps.

\section{Multiple Tasks}

We compared the results of Single-task (ST), Dual-task (DT) and Multi-task (MT). The results imply that the algorithm behaved similarly for ST and MT, but worse in DT especially in step direction detection (last row in Table VI). This corresponds with the fact that subjects made more mistakes in DT (21 out of 60 trials), compared with ST (7 out of 60 trials) and MT (9 out of 60 trials). The trials performed without mistakes are defined as the ones in which the subjects finished the test with 8 steps for each leg and in the correct direction. In a total of 60 trials of DT, 3 trials are with diagonal step and 1 trial is started with the wrong first step direction.

TABLE VI

ERROR DISTRIBUTION IN SINGLE-TASK, DUAL-TASK AND MULTI-TASK.

\begin{tabular}{lllll}
\hline & All & ST & DT & MT \\
\hline SEQ wrong & 41 & 13 & 13 & 15 \\
SEQ correct, RDIR or LDIR wrong & 10 & 1 & 8 & 1 \\
\hline
\end{tabular}

A further investigation in Table VII shows that when participants did not make mistakes, the algorithm succeeded $81.8 \%$ for SEQ and $79.7 \%$ for all the requirements. However, when the subjects made mistakes, the success rate decreased to $59.5 \%$ for SEQ and $40.5 \%$ for all the requirements.

TABLE VII

ALGORITHM PERFORMANCE WITH/WITHOUT SUBJECTS' MISTAKES.

\begin{tabular}{lccc}
\hline & All & SEQ & SEQ \& RDIR \& LDIR \\
\hline Without mistakes & 143 & $117(81.8 \%)$ & $114(79.7 \%)$ \\
With mistakes & 37 & $22(59.5 \%)$ & $15(40.5 \%)$ \\
\hline
\end{tabular}

\section{Auto check of detection failure}

As mention in Section III, the automatic check of detection failure has 5 false positives, which means 5 failed trials pass the check. After checking these 5 trials at the step level, we found that all of them failed at the beginning and/or end of the step sequence, for including extra steps outside the test period 
or losing the last steps at the end of data logging. Moreover, we found that all the directions of the steps within the test period were correct.

For the trials which pass the auto check of detection failure, their accuracy in step sequence detection is improved. In the trial level, the correct rate increases from $77.2 \%$ to $96.3 \%$. In the step level, the sensitivity increases from $99.3 \%$ to $99.9 \%$, and the PPV increases from $98.9 \%$ to $99.8 \%$.

Furthermore, the accuracy of step direction detection is improved. After the automatic check, all the 10 trials which fail in step direction detection are ruled out. Therefore, for the remaining trials, the correct rate of step direction detection improves from $92.8 \%$ to $100 \%$.

However, although auto verification can efficiently rule out the errors, it is recommended to manually segment the failed ones, especially when comparing different types of tests (e.g. ST, DT, and MT) or different groups of subjects. Checking only the trials which pass the auto-verification could lead to an overestimation on the performance of subjects because the trials without mistakes have a higher rate to be correctly segmented by the algorithm.

\section{CONCLUSion AND Future WORKS}

In this work, we proposed an algorithm that could automatically detect the step sequence and step direction during FSST, which had not been achieved to the knowledge of the authors. Experiments were conducted with healthy older adults in single-, dual-, and multi-task conditions. Manual verification with the video of the experiment was performed and auto verification method was developed, to check the results from the algorithm.

This work solved the two challenges for step segmentation in FSST. The first challenge, segmenting steps with various directions, is solved by identifying the static and non-static periods with the magnitude of foot angular velocity. The second challenge, segmenting multiple continuous steps of one leg, is solved by dividing the peaks in foot linear velocity.

The results showed that the algorithm succeeded for $71.7 \%$ trials in recognizing both the step sequence and step direction in FSST, while $90.2 \%$ of the detection failure can be automatically identified and then excluded from further data analysis by using the auto-verification method.

In the future, we will improve the correct rate of the proposed algorithm, especially when participants make mistakes. Furthermore, we plan to study the change in performance due to the cognitive load under dual- and multi-task conditions, and plan to compare with the young adults to reveal the change due to the aging process.

The proposed algorithm can be applied to other balance or functional mobility tests which require participants to step in different directions. Besides, it can be applied to the field of human activity recognition. Furthermore, with a little modification, the algorithm can be extended for teaching and assessment of dancing, especially the ballroom dancing.

\section{ACKNOWLEDGMENT}

The authors would like to express their thanks to the Italian Ministry of Foreign Affairs, General Directorate for Cultural
Promotion and Cooperation for its support to RoboCasa; Waseda University Program for Leading Graduate Schools; Tokyo Women's Medical University/Waseda University Joint Institution for Advanced Biomedical Sciences (TWIns). The authors would also like to express their gratitude to STMicroelectronics, Life Performance Research, SolidWorks Corp for their support to the research.

\section{REFERENCES}

[1] W. H. O. Ageing and L. C. Unit, WHO global report on falls prevention in older age. World Health Organization, 2008.

[2] M.-Y. Liaw, C.-L. Chen, Y.-C. Pei, C.-P. Leong, and Y.-C. Lau, "Comparison of the static and dynamic balance performance in young, middleaged, and elderly healthy people," Chang Gung Med J, vol. 32, no. 3, pp. 297-304, 2009.

[3] D. Sturnieks, R. St George, and S. Lord, "Balance disorders in the elderly," Neurophysiologie Clinique/Clinical Neurophysiology, vol. 38, no. 6, pp. 467-478, 2008.

[4] W. Dite and V. A. Temple, "A clinical test of stepping and change of direction to identify multiple falling older adults," Archives of physical medicine and rehabilitation, vol. 83, no. 11, pp. 1566-1571, 2002.

[5] W. Dite, H. J. Connor, and H. C. Curtis, "Clinical identification of multiple fall risk early after unilateral transtibial amputation," Archives of physical medicine and rehabilitation, vol. 88, no. 1, pp. 109-114, 2007.

[6] J. M. Blennerhassett and V. M. Jayalath, "The four square step test is a feasible and valid clinical test of dynamic standing balance for use in ambulant people poststroke," Archives of physical medicine and rehabilitation, vol. 89, no. 11, pp. 2156-2161, 2008.

[7] E. Y. Goh, S. Y. Chua, S.-J. Hong, and S. S. Ng, "Reliability and concurrent validity of four square step test scores in subjects with chronic stroke: a pilot study," Archives of physical medicine and rehabilitation, vol. 94, no. 7, pp. 1306-1311, 2013.

[8] R. P. Duncan and G. M. Earhart, "Four square step test performance in people with parkinson disease," Journal of Neurologic Physical Therapy, vol. 37 , no. 1 , pp. $2-8,2013$.

[9] S. L. Whitney, G. F. Marchetti, L. O. Morris, and P. J. Sparto, "The reliability and validity of the four square step test for people with balance deficits secondary to a vestibular disorder," Archives of physical medicine and rehabilitation, vol. 88, no. 1, pp. 99-104, 2007.

[10] X. Xu, R. W. McGorry, L.-S. Chou, J.-h. Lin, and C.-c. Chang, "Accuracy of the microsoft kinect for measuring gait parameters during treadmill walking," Gait \& posture, vol. 42, no. 2, pp. 145-151, 2015.

[11] M. Peterson, D. Ewins, A. Shaheen, and P. C. Formento, "Evaluation of methods based on conventional videography for detection of gait events," in VI Latin American Congress on Biomedical Engineering CLAIB 2014, Paraná, Argentina 29, 30 \& 31 October 2014, pp. 234-237, Springer, 2015.

[12] S. Sessa, K. Saito, M. Zecca, L. Bartolomeo, Z. Lin, S. Cosentino, H. Ishii, T. Ikai, and A. Takanishi, "Walking assessment in the phase space by using ultra-miniaturized Inertial Measurement Units," in 2013 IEEE International Conference on Mechatronics and Automation (ICMA), pp. 902-907, 2013.

[13] A. M. Sabatini, C. Martelloni, S. Scapellato, and F. Cavallo, "Assessment of walking features from foot inertial sensing," IEEE Transactions on biomedical engineering, vol. 52, no. 3, pp. 486-494, 2005.

[14] A. R. Jimenez, F. Seco, C. Prieto, and J. Guevara, "A comparison of pedestrian dead-reckoning algorithms using a low-cost mems imu," in Intelligent Signal Processing, 2009. WISP 2009. IEEE International Symposium on, pp. 37-42, IEEE, 2009.

[15] M. Ren, K. Pan, Y. Liu, H. Guo, X. Zhang, and P. Wang, "A novel pedestrian navigation algorithm for a foot-mounted inertial-sensor-based system," Sensors, vol. 16, no. 1, p. 139, 2016.

[16] H. Pohl and A. Hadjakos, "Dance pattern recognition using dynamic time warping," Sound and Music Computing, vol. 2010, 2010.

[17] P. R. Brustio, D. Magistro, E. Rabaglietti, and M. E. Liubicich, "Agerelated differences in dual task performance: A cross-sectional study on women," Geriatrics \& gerontology international, 2016.

[18] P. R. Brustio, D. Magistro, M. Zecca, M. E. Liubicich, and E. Rabaglietti, "Fear of falling and activities of daily living function: mediation effect of dual-task ability," Aging \& Mental Health, pp. 1-6, 2017. 